\title{
Intracranial Germ Cell Tumor in the Molecular Era
}

\author{
Ji Hoon Phi, M.D., Ph.D., Kyu-Chang Wang, M.D., Ph.D., Seung-Ki Kim, M.D., Ph.D. \\ Division of Pediatric Neurosurgery, Seoul National University Children's Hospital, Seoul National University College of Medicine, Seoul, \\ Korea
}

Intracranial germ cell tumors (iGCTs) are a heterogeneous group of tumors with peculiar characteristics clearly distinguished from other brain tumors of neuroepithelial origin. Diverse histology, similarity to gonadal GCT, predilection to one sex, and geographic difference in incidence all present enigmas and fascinating challenges. The treatment of iGCT has advanced for germinoma to date; thus, clinical attention has shifted from survival to long-term quality of life. However, for non-germinomatous GCT, current protocols provide only modest improvement and more innovative therapies are needed. Recently, next-generation sequencing studies have revealed the genomic landscape of iGCT. Novel mutations in the KIT-RAS-MAPK and AKT-MTOR pathways were identified. More importantly, methylation profiling revealed a new method to assess the pathogenesis of iGCT. Molecular research will unleash new knowledge on the origin of iGCT and solve the many mysteries that have lingered on this peculiar neoplasm for a long time.

Key Words : Germ cell · Germinoma · Mutation · Methylation.

\section{INTRODUCTION}

Intracranial germ cell tumors (iGCTs) are a group of brain tumors with extraordinary characteristics. The cardinal features of brain tumors, such as age of onset, tumor location, histopathology and biological behavior, are quite distinct from other brain tumors of neuroepithelial origins. However, studies on molecular pathogenesis of iGCT lag behind the achievements noted for other brain tumors. The relatively low incidence and more importantly striking geographical difference of incidence precluded clinical interest and prevented global collaborative research for iGCT. Heterogeneity in patient ages and multiple pathological subgroups serve as a source of confusion between clinicians and researchers. Furthermore, the paradigm shift from radical surgery to biopsy with adjuvant therapies provided less tumor tissues for research than previously available. Despite these limitations, progress has been made in iGCT research, and our knowledge of the pathogenesis of iGCT has increased. In this review, we will present at overview current clinical knowledge on iGCT. Then, we will discuss the molecular pathogenesis of iGCT based on recent advancements.

\section{EPIDEMIOLOGY OF IGCT}

iGCT is histologically identical to GCT developing in other parts of the body. The majority of GCT cases arise from the

- Received : March 12, 2018 •Revised : April 2, 2018 •Accepted : April 16, 2018

- Address for reprints : Seung-Ki Kim, M.D., Ph.D.

Division of Pediatric Neurosurgery, Seoul National University Children's Hospital, Seoul National University College of Medicine, 101 Daehak-ro, Jongno-gu, Seoul 03080, Korea

Tel : +82-2-2072-2350, Fax : +82-2-744-8459, E-mail : nsthomas@snu.ac.kr

This is an Open Access article distributed under the terms of the Creative Commons Attribution Non-Commercial License (http://creativecommons.org/licenses/by-nc/4.0) which permits unrestricted non-commercial use, distribution, and reproduction in any medium, provided the original work is properly cited. 
gonads, i.e., testis and ovary. Therefore, a considerable amount of iGCT knowledge, such as histological classification, tumor markers, and even chemotherapeutic regimens, stemmed from the clinical experience of gonadal GCTs. iGCT is a special group of extragonadal GCTs that develops outside gonads. The most common sites of extragonadal GCTs include brain, mediastinum, retroperitoneum, and sacrococcyx ${ }^{38)}$. Interestingly, all these sites are lined along the midline of the body, and many hypotheses have been proposed for this phenomenon. Even in the brain, iGCT develops in midline structures. Pineal and suprasellar regions are the most common sites for iGCT. Occasionally, iGCT is found at both sites simultaneously (so-called bifocal GCTs). Approximately 10\% of iGCT arise from the basal ganglia, which is slightly off the midline. However, basal ganglia can be considered as midline structures separated by a narrow slit (the third ventricle). It is also noteworthy that all these locations are situated around the third ventricle. iGCT is rarely found in the cerebral hemisphere, cerebellum and spinal cord.

The striking geographical difference of iGCT incidence is interesting. iGCT is far more frequently diagnosed in East Asian countries, especially Korea and Japan. In the Korean Central Cancer Registry, the incidence of IGCT is 3.4/million/ year $^{11)}$. The reported incidence is $2.7 /$ million/year in Japan and $0.6 /$ million/year in the USA ${ }^{32}$. In contrast, gonadal GCT, especially malignant testicular GCT, is much more common in Caucasians in Western countries (12/10000/year in Denmark, Norway, and Switzerland) compared with East Asia (2/10000/year in Japan $)^{56)}$. Malignant ovarian GCT occurs less frequently than testicular GCT, and no geographical difference is noted ${ }^{56)}$. The difference in iGCT incidence according to sex is also interesting. In all countries, iGCT is more common in males. In Korea, the male-to-female incidence ratio is $4.53: 1^{11)}$. In USA, the maleto-female ratio is $3.9: 1^{29)}$. The male predominance of iGCT is more profound for pineal tumors compared with suprasellar tumors (13.0: 1 and 1.73: 1 in USA) ${ }^{18)}$. Mediastinal GCT is also more common in males than in females, but sacrococcygeal GCT is more common in females ${ }^{56)}$.

Two age peaks are noted in iGCT incidence. A small peak exists for infants (0-2 years) and large peak stands for adolescents (13-19 years). Incidence declines rapidly after young adulthood. However, in a broader perspective including both gonadal and extragonadal GCTs, GCT appears to have three age peaks : infants, adolescents, and elderly. Infants mainly develop extragonadal GCTs, mostly in the brain and sacrococcyx. The majority of these infantile GCTs are teratoma (TE) and yolk sac tumor $(\mathrm{YST})^{38)}$. Infantile GCT is more common in females. In adolescents, both gonadal and extragonadal GCTs are increased. Seminoma (gonadal) and germinoma (GE) (extragonadal) are major pathologies. In elderly individuals, gonadal (testicular) GCT increases as a form of spermatocytic seminoma. Infantile brain TE/YSTs are typically large tumors situated in the third ventricle and are often diagnosed in utero with accompanying hydrocephalus. Extirpation of large brain tumors in the neonatal period presents serious challenges to surgeons and physicians. Infantile mature TE can recur as immature TE or YST when incompletely resected ${ }^{39,60)}$. In adolescents, iGCT starts to develop around the onset of puberty. Therefore, hormonal influence is strongly suspected for iGCT development in adolescents and young adults.

\section{HISTOPATHOLOGICAL SUBGROUP}

As gonadal GCTs, iGCT is divided into two broad categories : GE and non-germinomatous GCT (NGGCT). NGGCT is further subdivided into four subgroups : TE, YST, choriocarcinoma (ChC), and embryonal carcinoma (EC). The distinction of GE from NGGCT is derived from the original theory of GCT pathogenesis, the so-called 'germ cell theory' proposed by Teilum $^{54)}$. In germ cell theory, GCT originates from primordial germ cells through neoplastic transformation. Each NGGCT arises from more differentiated stages of embryonic development starting from germ cells. Therefore, GE is a prototype of all GCTs. NGGCTs develop from more differentiated counterparts of embryonic and extraembryonic tissues.

The dualistic distinction of GE/NGGCT has more practical connotations than theoretical arguments. Although intracranial GE is a malignant tumor that spreads readily in the ventricles, GE is highly sensitive to radiation therapy (RT) and chemotherapy. GE is not considered a surgical disease. Therefore, biopsy followed by RT and chemotherapy is a standard protocol for GE. On the other hand, surgery plays a more important role for NGGCTs. Especially, TE is typically unresponsive to RT and chemotherapy, and surgery is the only therapeutic option for the disease. The prognosis of GE is excellent with $>95 \%$ longterm survival. With the exception of benign mature TE (95$100 \%$ survival), NGGCT generally exhibits a poor prognosis compared with GE. Actually, the prognosis of YST and ChC was 
considered dismal until very recently. Therefore, from a clinical viewpoint, distinguishing GE from NGGCT is practical and highly recommended. In 1997, Dr. Matsutani of Japan proposed a scheme of prognosis groups of iGCTs, based on long-term treatment outcome ${ }^{28}$. GE and mature TE comprise the good prognosis group. Immature/malignant TE and mixed GCT consisting of GE/TE belong to the intermediate prognosis group. Highly malignant NGGCTs are included in the poor prognosis group. This scheme has been widely used in clinical practice. In Europe and North America, a dichotomous scheme of GE and NGGCT has been favored ${ }^{31}$. More specifically, division between tumor marker-positive and marker-negative iGCT is considered useful. In the European SIOP-CNS-GCT-96 trial, serum/ cerebrospinal fluid (CSF) alpha fetoprotein (AFP) $>1000 \mathrm{ng} / \mathrm{mL}$ was identified as a poor prognostic factor ${ }^{6}$.

\section{THE ENIGMA OF MIXED GCT}

A confounding issue for iGCT subgrouping is the presence of mixed GCT. Mixed GCT has multiple components of individual iGCT subgroups. All mixed GCT logically belong to NGGCT. GE and TE are the most common components of mixed GCT. If malignant components, such as EC, YST, or ChC, are observed, the grade of the entire tumor is escalated to the poor prognosis group. Approximately $10-30 \%$ of iGCT are mixed GCT, but the proportion considerably increases if we search the entire paraffin block of pathological specimens for the trace of other components. If an iGCT mass is composed of 99\% YST and $1 \%$ GE, can we call it mixed GCT or YST? If we miss the tiny $1 \%$ of GE in pathological diagnosis or the bit is not even included in surgical biopsy specimen, the diagnosis will be pure (100\%) YST. It is not certain whether pure YST and 99\% YST (+1\% GE) are different diseases. However, small components in mixed GCT may be important in some instances. TE components in malignant mixed GCT can survive RT and chemotherapy. Paradoxical tumor growth is occasionally observed during or after adjuvant therapies. In this so-called 'growing teratoma syndrome', mature or immature TE grows rapidly to an enormous size, causing a mass effect and hydrocephalus ${ }^{25)}$. Early detection and surgical removal are critical for treatment.

\section{DIAGNOSIS}

Typical age of onset, sex, symptoms and image characteristics make the diagnosis straightforward in many instances. However, without clinical suspicion, it is occasionally very difficult to diagnose iGCT. Patients with suprasellar GCT typically present with diabetes insipidus that persists for months and even years. Loss of normal bright signal intensity in the posterior hypophysis is a characteristic finding ${ }^{42)}$. Growth retardation and short statue are also common. If a tumor becomes large, visual disturbance and hydrocephalus can develop. Pineal GCT typically present with symptoms of hydrocephalus by obstruction of the cerebral aqueduct. Common symptoms and signs include headache, nausea, vomiting, visual disturbance, and abducens nerve palsy. Parinaud's syndrome with a classic triad of upward gaze palsy, light-near dissociation, and convergence-retraction nystagmus can be observed. Precocious puberty is occasionally found in patients with suprasellar or pineal $\mathrm{GCT}^{42)}$. Beta human chorionic gonadotrophin ( $\beta$-HCG) secreted by GE and ChC components is associated with this phenomenon. Patients with basal ganglia GCT typically present with slowly progressive hemiparesis. Muscle atrophy and contracture are common but somatosensory function is preserved. The symptoms are so insidious that misdiagnosis is frequent. Atrophy of the ipsilateral pyramidal tract in medulla oblongata, cerebral peduncle, caudate nucleus, or cerebral hemisphere is a frequent finding on magnetic resonance imaging ${ }^{40)}$.

GE, especially of suprasellar origin, tends to be occult with invisible or very small lesion causing diabetes insipidus (socalled occult suprasellar GE) ${ }^{23,30}$. Basal ganglia GE sometimes exhibit similar insidious clinical course. The long latency between symptom onset and overt tumor progression of GE is an enigma and hormonal influence after puberty is suspected. Bifocal tumor involving both suprasellar and pineal areas constitute approximately $6-41 \%$ of iGCT ${ }^{41)}$. Bifocal presentation has been regarded as a pathognomonic sign of GE, but some of the patients actually have mixed $\mathrm{GCT}^{41}$. It is not clear whether bifocal GCT is metastatic spread from one site or synchronous development. Ventricular seeding is common for GE, but diffuse leptomeningeal seeding over the cerebral cortex or spinal subarachnoid space is relatively uncommon. Therefore, the RT field should routinely include whole ventricles rather than limited to the tumor mass ${ }^{31}$. Craniospinal RT is indicated for patients with evidence of diffuse leptomeningeal seeding. 
The presence of serum and CSF tumor markers is a unique characteristic of iGCT. $\beta$-HCG is markedly elevated in $\mathrm{ChC}$, often greater than 10000 IU/L. $\beta$-HCG levels may be elevated in some patients with EC, immature teratoma, and GE. Increased serum/CSF $\beta$-HCG in GE is attributed to the presence of syncytiotrophoblasts but the possibility of mixed GCT should be considered. An arbitrary value of 50-100 IU/L is used to distinguish GE with syncytiotrophoblasts and mixed GCT with ChC components. The prognostic value of mild elevation of the $\beta$-HCG in GE is controversial. AFP is typically increased in YST, but its level is also elevated in some of EC and immature $\mathrm{TE}^{43)}$. Usually, the $\beta$-HCG level is higher in CSF compared with serum, and the AFP level is increased in serum compared with CSF. Therefore, for the diagnosis of occult GE, assessment of CSF $\beta$-HCG is recommended ${ }^{30)}$. However, tumor marker measurement methods are not standardized and cutoff values for diagnosis and risk stratification are not clearly defined.

\section{TREATMENT}

The treatment of iGCT should be multidisciplinary, incorporating surgery, chemotherapy, RT, and endocrine therapy. The major role of surgery is the acute management of hydrocephalus that frequently accompanies iGCT and tissue biopsy for diagnosis. With endoscopic procedures, neurosurgeons can achieve both goals in a single operation for suprasellar and pineal GCT. For basal ganglia GCT, a stereotactic biopsy is usually applied.

Treatment decision follows serum/CSF tumor marker expression. Marker-negative iGCT, that is, a tumor without elevated serum/CSF $\beta$-HCG and AFP, indicates GE or TE. Imaging can distinguish the two entities in many cases, but surgical biopsy is frequently undertaken to confirm the diagnosis. For mature TE, gross total resection provides disease cure. However, immature TE frequently requires adjuvant therapies ${ }^{43)}$. Surgery has a limited role for GE except for biopsy and diagnosis. RT is the mainstay of treatment of GE. Previously, 36 Gy craniospinal RT with a tumor boost of $15 \mathrm{~Gy}$ was the standard treatment of GE, which yielded excellent outcome ( $>90 \%$ long-term survival ${ }^{46)}$. However, radiation can cause long-term sequelae of endocrinopathy, short statue, cognitive decline, and secondary malignancy. Therefore, RT fields and radiation doses have been reduced for GE. In a review of published data, whole brain or whole ventricular RT resulted in slightly more relapse (7.6\%) than craniospinal RT with tumor boost (3.8\%), but the difference was not statistically significant ${ }^{46)}$. Focal RT should be avoided because it yielded an increased relapse rate (23.3\%). Currently, localized GE is treated by whole brain or whole ventricular RT, with tumor doses of 36-39 Gy and whole ventricular doses of 19.5-24 Gy ${ }^{8,935)}$. A short course of chemotherapy can be added before RT. The tumor outcome is excellent with $>95 \%$ longterm survival. For disseminated GE, craniospinal RT is required.

Pre-radiation chemotherapy is commonly applied for GE. The aim of chemotherapy is not to enhance survival (survival rate is high enough with RT) but to reduce RT doses and potential complications of irradiation. The recently published SIOPCNS-GCT 96 trial demonstrated that chemotherapy followed by reduced volume and dose RT yielded comparable outcome with craniospinal RT alone ${ }^{7)}$. Chemotherapy alone for GE has been attempted, but high rates of failure preclude this approach ${ }^{24)}$.

The prognosis of marker-positive iGCT is worse than that of GE and TE. Nearly all marker-positive iGCT are NGGCT except for GE with a mildly high level of $\beta$-HCG $(<50-100$ IU/L). Surgical resection, intensive chemotherapy, and craniospinal RT should be applied to treat malignant NGGCT. In 1990s, the reported 5-year-survival rate of malignant NGGCTs was approximately $30 \%{ }^{28,49)}$. Intensified multimodality treatment greatly enhanced the outcome of NGGCT. In recent retrospective studies on NGGCT, long-term survival rates of 74-80\% were reported $^{26,27)}$. However, NGGCT is a very heterogeneous group of diseases with different prognoses. For example, mixed GCT mainly composed of GE plus immature TE definitely has better prognosis than pure YST or ChC. This heterogeneity makes comparison of outcomes from different studies, patient cohorts, and trials difficult. Recent trends show that the role of surgical resection for NGGCT is reduced. In the German MAKEI89 trial, surgery was not a significant factor for treatment outcome, but craniospinal RT and higher cisplatin dose were important ${ }^{5)}$. Current standard for NGGCT is chemotherapy to shrink the tumor volume followed by craniospinal RT. The goal of chemotherapy for NGGCT is not to reduce radiation volume and dose, but to maximize the likelihood of survival ${ }^{31)}$. During or after chemotherapy and RT, paradoxical growth of tumors (growing teratoma syndrome) should be treated by surgery. Second-look surgery is also considered for residual lesions after RT is complete. Published studies demonstrated that the majority of residual lesions were TE or scar tissues ${ }^{36,58)}$. However, the indication and timing of second-look surgery are not established yet. 


\section{THEORIES ON CELLS OF ORIGIN FOR IGCT}

There are diverse theories about the cells of origin for iGCT. These theories can be largely divided into two major streams often with minor modifications. The first one is traditional 'germ cell theory'. This theory dictates that gonadal GCT originates from primordial germ cells (PGC) through transformation. The development of extragonadal GCT is explained by the presence of ectopic germ cells that deviate from fetal PGC migration. In many animals, including humans, PGCs arising from the yolk sac epithelium are separated with somatic gonadal tissues and therefore should migrate to gonadal areas ${ }^{45)}$. This migration process can give rise to ectopic germ cells in the midline of the body. GE is the neoplastic counterpart of PGCs or slightly more committed gonocytes. NGGCTs can develop from transformed PGCs/ gonocytes through epigenetic reprogramming ${ }^{38)}$. Thus, in germ cell theory, all iGCTs, including GE and NGGCT are truly germ cell tumors. The second theory is the so-called 'pluripotent stem cell theory'. In this theory, seminoma/GE may originate from PGCs/gonocytes. NGGCT develops from embryonic stem (ES) cells with pluripotent potentials. EC is the prototype of all NGGCTs, which is the neoplastic counterpart of ES cells. YST, ChC, and TE can develop from the transformed EC cells. Extragonadal GCT can develop in a similar pattern from tissueresiding pluripotent stem cells through neoplastic transformation. The experimental fact that ES cells can give birth to germ cells leads to a suggestion that seminoma/GE may also develop from ES cells through the stage of PGCs/gonocytes. However, it cannot be settled by current evidence whether pluripotent stem cells are truly remnant ES cells (or ES-like cells) or reprogrammed PGCs.

\section{SEMINOMA/GE ORIGINATES FROM GERM CELLS}

Germ cell origin of seminoma/GE is supported by multiple lines of evidence. First, the hypothesis was derived from the morphological resemblance of seminoma/GE cells to PGCs. The PGC is characterized by its large size and plump and round nucleus with conspicuous nucleolus, which are also typical features of seminoma/GE cells ${ }^{12)}$. Second, seminoma/GE express pluripotency markers such as OCT4, NANOG, and SOX17, that are expressed by PGCs and ES cells ${ }^{10,15)}$. Seminoma/GE also strongly express PLAP and KIT ${ }^{33)}$ (Fig. 1). PLAP and KIT represent markers of germ cell lineage differentiation derived from ES cells. Therefore, protein expression patterns support germ cell origin of the tumors. Third, PGCs undergo distinct changes of DNA methylation and demethylation during embryonic development. PGCs exhibit global erasure of methylation marks. PGCs gradually acquire methylation and sex-specific imprinting patterns during gametogenesis ${ }^{21)}$. In testicular GCTs, seminoma exhibits global promotor hypomethylation with erasure of imprinting marks. The methylation status of seminoma closely resembles that of $\mathrm{PGCs}^{34)}$. GE also exhibit similar global hypomethylation, supporting germ cell origin of seminoma/ $\mathrm{GE}^{14)}$. Fourth, PGCs are highly dependent on KIT signaling. KIT is a receptor tyrosine kinase and is crucial in the survival, prolifer-
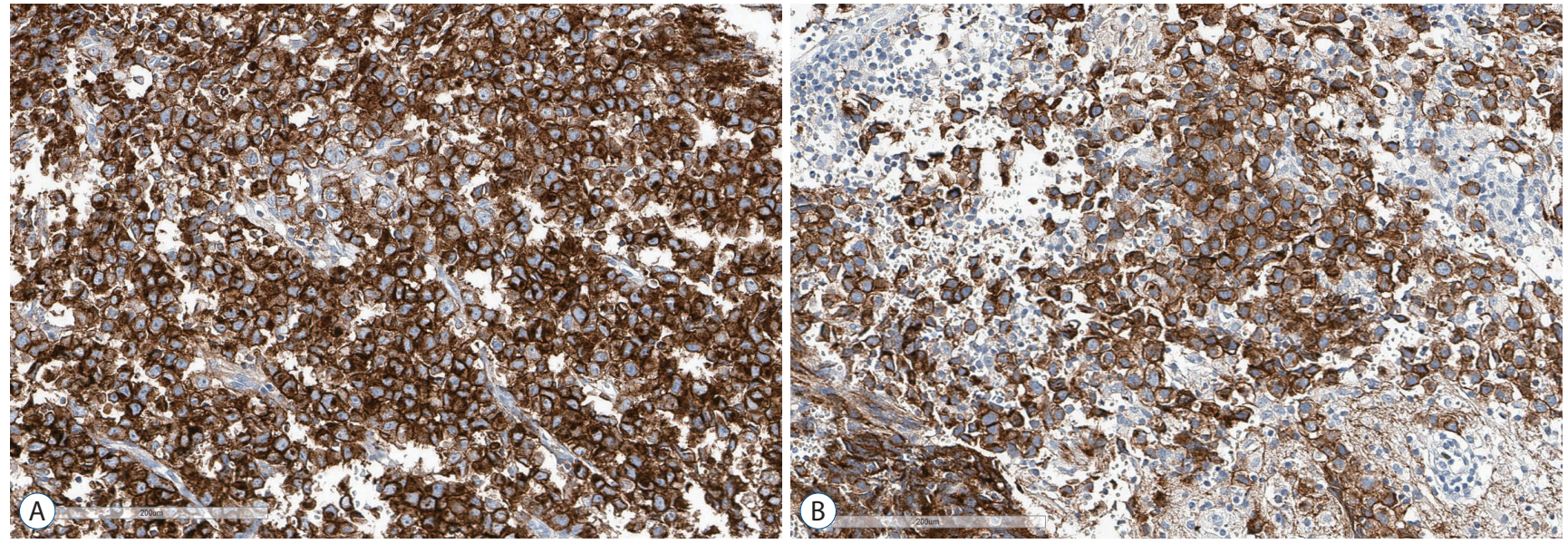

Fig. 1. Strong (A) PLAP and (B) KIT expression in GE cells (immunohistochemistry, $\times 200$ ). PLAP: placental alkaline phosphatase, GE: germinoma. 
ation, and migration of PGCs ${ }^{50)}$. Its ligand, KITLG (also known as stem cell factor [SCF]) is provided by stromal cells of gonads ${ }^{19)}$. KIT signaling is upstream of RAS-MAPK signaling and PI3K pathway, which are involved in a variety of cellular processes. Activating mutations in KIT and other genes in MAPK and PI3K pathways are the most common genomic variations found in both seminoma and $\mathrm{GE}^{17,20)}$. KIT signaling is activated in seminoma/GE, reflecting PGCs as their cell of origin.

\section{NGGCT MAY ORIGINATE FROM PLURIPOTENT STEM CELLS}

As for gonadal and extragonadal NGGCT, the cell of origin problem is less clear than GE. In traditional germ cell theory, neoplastic germ cell (i.e., seminoma/GE) can evolve into more differentiated tissue-like tumors : YST resembling endodermal sinus, ChC similar to trophectoderm, and TE representing ectoderm, mesoderm and endoderm. However, the germ cell is not pluripotent. Germ cells need reprogramming to unchain its pluripotency potentials $^{38)}$. In the 1990s, Dr. Sano raised an intriguing question, namely, why more differentiated NGGCTs have worse prognosis than more primitive GE in Teilum's hier$\operatorname{archy}^{48)}$. Actually, cancers of undifferentiated histology occasionally exhibit better prognosis because RT and chemotherapy are more effective for rapidly proliferating, undifferentiated cells. Nonetheless, this query led to the suggestion that NGGCT may have different cells of origin from GE. The most plausible candidates are pluripotent stem cells residing in gonadal and extragonadal tissues.

In the 1970s, it was discovered that EC cells exhibit pluripotency. The research on EC cell pluripotency lead to the isolation of ES cells of mice in $1980 \mathrm{~s}^{3)}$. Thus, EC cells are regarded as neoplastic counterparts of ES cells. The robust expression of pluripotency markers, including OCT4, NANOG, and SOX2, in EC supports this conjecture ${ }^{16)}$. Therefore, EC is considered to originate from neoplastic pluripotent stem cells. Then, more differentiated subtypes such as YST, ChC, and TE evolve from pluripotent stem cells with or without an intervening form of EC. Oosterhuis et al. ${ }^{38)}$ suggested dual cell of origins for NGGCTs. Infantile GCT, all of which are YST or TE, are close to ES cells, displaying partially erased imprinting marks, whereas post-pubertal NGGCT exhibit more completely erased imprinting patterns, resembling germ cells ${ }^{38)}$. However, in testicular GCT, the genome of seminoma exhibits global hypomethylation, and all NGGCT subtypes have global hypermethylation similar to somatic tissues ${ }^{34)}$. A recent large-scale methylation profiling of iGCT reveals that iGCT exhibits similar methylation patterns as testicular GCT : global hypomethylation in GE and hypermethylation in $\mathrm{NGGCT}^{14)}$. If GCT retains methylation patterns after neoplastic transformation from the cell of origin, NGGCT does not seem to originate from PGCs/gonocytes but from more primitive stem cells-that are close to ES cells. The histological diversity of NGGCT can be attributed to the pluripotency of these stem cells.

\section{THE GENOMIC LANDSCAPE OF IGCT}

The importance of KIT signaling in germ cells led to interests in this proto-oncogene in testicular GCT. Earlier reports indicated high expression of c-KIT in seminoma but not in NGGCT. Activating KIT mutations are found in seminoma, especially in bilateral cases ${ }^{4,55)}$. Studies have demonstrated that up to $25 \%$ of seminoma has mutations in KIT or KRAS genes, which are mutually exclusive. However, these mutations are rare in NGGCT ${ }^{2,51)}$. In early studies before the era of next-generation sequencing, KIT mutation was also found in 25\% of germino$\mathrm{ma}^{22,47)}$. Interestingly, the most characteristic genetic event in testicular GCT is a gain of chromosome 12p, which is observed in almost all testicular GCTs. Isochromosome $12 \mathrm{p}[\mathrm{i}(12 \mathrm{p})]$ is the most frequent form (80\%) and the remaining cases harbor duplication or focal amplification of $12 \mathrm{p}^{44)} . \mathrm{i}(12 \mathrm{p})$ is not present in infantile GCT and spermatocytic seminoma in elderly. Many genes on chromosome $12 \mathrm{p}$ have been implicated as driver oncogenes, i.e., KITLG, NANOG, KRAS, BCAT1, and CCND2, but none have been definitely proven ${ }^{51)}$. At present, gain of chromosome $12 \mathrm{p}$ combined with activated KIT signaling appear to be the key molecular trigger in gonadal GCT pathogenesis.

However, gain of chromosome $12 \mathrm{p}$ material is less frequently observed in iGCTs. A study reported only $20 \%$ of iGCT had increased $12 \mathrm{p}$ including $\mathrm{i}(12 \mathrm{p})$. The other study indicated that $25 \%$ of iGCT harbored i(12p) and 46\% had polyploidy of chromosome $12^{37,52)}$. The discrepancy reflects small numbers of cases in each study, but it is clear that chromosome $12 \mathrm{p}$ gain plays a less crucial role in iGCT compared with testicular GCT. The other frequent chromosomal abnormality involve gain of $\mathrm{X}$, 21q, and 14q. It is noteworthy that Kleinfelter syndrome (46, XXY) 
and Down syndrome $(47,+21)$ are associated with extragonadal GCT including iGCT ${ }^{1,53)}$.

Recently, whole exome sequencing of iGCT tissues reveals mutations in KIT (26\%), KRAS/NRAS (20\%), CBL (11\%), MTOR $(8 \%)$, and NF1 $(3 \%)^{57)}$. KRAS/NRAS constitutes the downstream pathway of KIT receptor tyrosine kinase, feeding into MAPK pathways. Activating KIT and KRAS/NRAS mutations are mutually exclusive. CBL is a negative regulator of KIT-RAS signaling. NF1 is another negative regulator of RAS-MAPK pathway. A separate downstream pathway of KIT receptor consists of AKT1 and MTOR. Amplification of AKT1 is also observed in 19\%. Overall, 53\% of iGCT have one or more of genetic variations in the KIT-RAS-MAPK or AKT-MTOR pathways (Fig. 2). Interestingly, these genetic variations occur predominantly in GE. The same mutations are infrequently found in NGGCT. In another study on genomic features of iGCT, KIT mutation was found in $40 \%$ of GE and $6 \%$ of $\mathrm{NGGCT}^{13)}$. RAS mutations were observed in 20\% of GE and 3\% of NGGCT. Many NGGCT cases with KIT/RAS mutations were actually mixed GCTs. Therefore, it is likely that, unlike GE, intracranial pure NGGCTs (i.e., pure EC, YST, ChC, and TE) are less dependent on KIT/RAS signaling.

An interesting study demonstrated that micro-dissected GE and NGGCT components of mixed GCT share the common KIT/RAS mutations, but differ in global methylation profile :

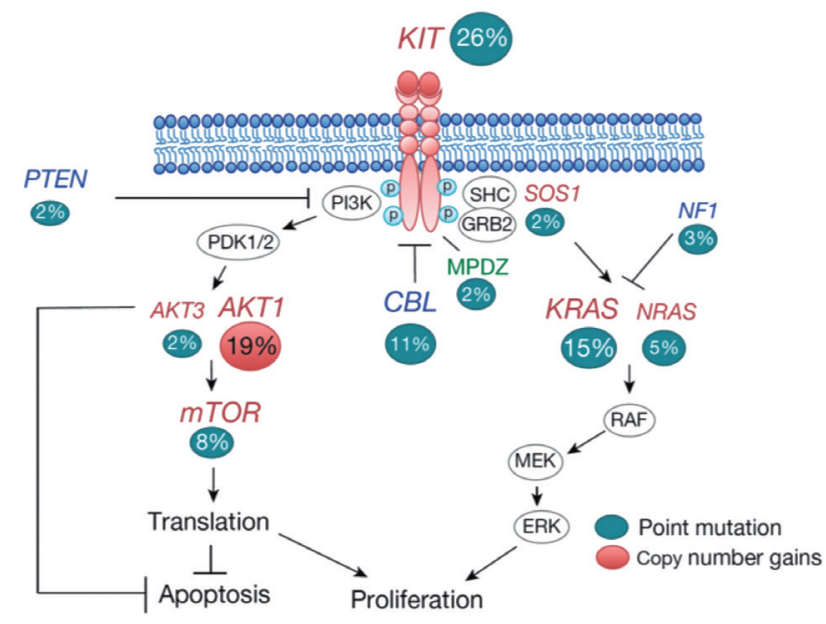

Fig. 2. Genetic alterations in KIT-RAS-MAPK and AKT-MTOR pathways in iGCTs. The iGCTs include 29 GE and 33 NGGCT including 8 mixed GCT. Red text, protein positively regulates signalling; blue text, protein negatively regulates signalling; green text, physically interacting protein. Reprint from Wang et al. ${ }^{57)}$ with permission from Springer Nature. iGCT : intracranial germ cell tumors, NGGCT : non-germinomatous GCT. hypomethylation in GE components and hypermethylation in NGGCT components ${ }^{14)}$. Therefore, mixed GCT may develop from the same cell of origin, presumably PGCs with KIT/RAS mutation as an initiating event. Then, NGGCT components can be derived from GE through epigenetic reprogramming.

iGCT is characterized by peculiar geographic, age, and sex predilections. Genetic susceptibility has been suspected to explain the epidemiological features of iGCT. A rare germline variant of JMJD1C gene is enriched in iGCT patients, especially in Japanese patients ${ }^{57)}$. The variant (S880P) is also enriched in the Japanese population. JMJD1C is a chromatin modifier and acts in germline development. More importantly, JMJD1C interacts with androgen receptor $(\mathrm{AR})^{59)}$. Through interaction with $\mathrm{AR}$, the rare polymorphism of JMJD1C may account for the increased incidence in East Asia and male predominance of iGCT.

\section{PERSPECTIVES}

iGCT has attracted the interests of clinicians and researchers given the diverse histology, similarity to gonadal GCT, unusual epidemiological facts, and mysterious pathogenesis. From a clinical viewpoint, iGCTs are malignant brain tumors with the best prognosis currently known to neuro-oncologists. For GE, the treatment focus is shifting from survival to quality of life. RT dose reduction, endocrinological therapy, and psychosocial support are the main focuses of interest. For NGGCT, more promising outcomes are being unleashed with multimodal therapies. Actually, iGCT can be an index disease where surgery, chemotherapy, and RT all contribute greatly and effectively to enhance patients' outcomes. Recent advancements in genomewide analysis reveal very interesting findings regarding underlying genetic mutations, altered signaling, and most importantly methylation profiling. This research can lead us not only to a new hypothesis of iGCT pathogenesis but also to novel therapy targeting the aberrant signaling pathways. KIT mutations are commonly found in chronic myeloid leukemia and gastrointestinal stromal tumors. Tyrosine kinase inhibitors, such as imatinib mesylate and dasatinib showed fair efficacy against these KIT-activated human malignancies. Although the prognosis of GE is excellent by current protocols, some patients develop recurrence. These patients can be salvaged by tyrosine kinase inhibitors in the future. Furthermore, a novel therapy is highly requested for NGGCT, and more studies are needed to define the 
therapeutic targets in NGGCT.

\section{CONFLICTS OF INTEREST}

No potential conflict of interest relevant to this article was reported.

\section{INFORMED CONSENT}

This type of study does not require informed consent.

\section{- Acknowledgements}

This work (2017R1A2B2008422) was supported by Mid-career Researcher Program through NRF grant funded by the Korea government (Minstry of Science and ICT).

\section{References}

1. Aguirre D, Nieto K, Lazos M, Peña YR, Palma I, Kofman-Alfaro S, et al. : Extragonadal germ cell tumors are often associated with Klinefelter syndrome. Hum Pathol 37 : 477-480, 2006

2. Bamford S, Dawson E, Forbes $S$, Clements J, Pettett R, Dogan A, et al. : The COSMIC (Catalogue of Somatic Mutations in Cancer) database and website. Br J Cancer 91 : 355-358, 2004

3. Barbaric I, Harrison NJ : Rediscovering pluripotency: from teratocarcinomas to embryonic stem cells. Cardiff, 10-12 October 2011. Int J Dev Biol 56 : 197-206, 2012

4. Biermann $K$, Klingmuller $D$, Koch $A$, Pietsch $T$, Schorle $H$, Büttner $R$, et al. : Diagnostic value of markers M2A, OCT3/4, AP-2gamma, PLAP and c-KIT in the detection of extragonadal seminomas. Histopathology 49 : 290297, 2006

5. Calaminus $G$, Bamberg $M$, Jurgens $H$, Kortmann RD, Sörensen N, Wiestler $\mathrm{OD}$, et al. : Impact of surgery, chemotherapy and irradiation on long term outcome of intracranial malignant non-germinomatous germ cell tumors: results of the German Cooperative Trial MAKEl 89. Klin Padiatr 216 : 141-149, 2004

6. Calaminus G, Frappaz D, Kortmann RD, Krefeld B, Saran F, Pietsch T, et al. : Outcome of patients with intracranial non-germinomatous germ cell tumors-lessons from the SIOP-CNS-GCT-96 trial. Neuro Oncol 19 : 1661-1672, 2017

7. Calaminus G, Kortmann R, Worch J, Nicholson JC, Alapetite C, Garrè ML, et al. : SIOP CNS GCT 96: final report of outcome of a prospective, multinational nonrandomized trial for children and adults with intracrania germinoma, comparing craniospinal irradiation alone with chemotherapy followed by focal primary site irradiation for patients with localized disease. Neuro Oncol 15 : 788-796, 2013

8. Cheng S, Kilday JP, Laperriere N, Janzen L, Drake J, Bouffet E, et al. : Outcomes of children with central nervous system germinoma treated with multi-agent chemotherapy followed by reduced radiation. J Neurooncol $127: 173-180,2016$

9. Cho J, Choi JU, Kim DS, Suh CO : Low-dose craniospinal irradiation as a definitive treatment for intracranial germinoma. Radiother Oncol 91 : 75-79, 2009

10. de Jong J, Stoop H, Gillis AJ, van Gurp RJ, van de Geijn GJ, de Boer M, et al. : Differential expression of SOX17 and SOX2 in germ cells and stem cells has biological and clinical implications. J Pathol 215 : 21-30, 2008

11. Dho YS, Jung KW, Ha J, Seo Y, Park CK, Won YJ, et al. : An updated nationwide epidemiology of primary brain tumors in Republic of Korea, 2013. Brain Tumor Res Treat 5 : 16-23, 2017

12. Fujimoto $\mathrm{T}$, Miyayama $\mathrm{Y}$, Fuyuta $\mathrm{M}$ : The origin, migration and fine morphology of human primordial germ cells. Anat Rec 188 : 315-330, 1977

13. Fukushima S, Otsuka A, Suzuki T, Yanagisawa T, Mishima K, Mukasa A, et al. : Mutually exclusive mutations of KIT and RAS are associated with KIT mRNA expression and chromosomal instability in primary intracranial pure germinomas. Acta Neuropathol 127 : 911-925, 2014

14. Fukushima S, Yamashita S, Kobayashi H, Takami H, Fukuoka K, Nakamura T, et al. : Genome-wide methylation profiles in primary intracranial germ cell tumors indicate a primordial germ cell origin for germinomas. Acta Neuropathol 133 : 445-462, 2017

15. Gashaw I, Dushaj O, Behr R, Biermann K, Brehm R, Rübben H, et al. : Novel germ cell markers characterize testicular seminoma and fetal testis. Mol Hum Reprod 13 : 721-727, 2007

16. Gillis AJ, Stoop H, Biermann K, van Gurp RJ, Swartzman E, Cribbes S, et al. : Expression and interdependencies of pluripotency factors LIN28, OCT3/4, NANOG and SOX2 in human testicular germ cells and tumours of the testis. Int J Androl 34(4 Pt 2) : e160-e174, 2011

17. Goddard NC, McIntyre A, Summersgill B, Gilbert D, Kitazawa S, Shipley $\mathrm{J}:$ KIT and RAS signalling pathways in testicular germ cell tumours: new data and a review of the literature. Int J Androl 30 : 337-348; discussion 349, 2007

18. Goodwin TL, Sainani K, Fisher PG : Incidence patterns of central nervous system germ cell tumors: a SEER study. J Pediatr Hematol Oncol 31 : 541-544, 2009

19. Høyer PE, Byskov AG, Møllgård K : Stem cell factor and c-Kit in human primordial germ cells and fetal ovaries. Mol Cell Endocrinol 234 : 1-10, 2005

20. Ichimura K, Fukushima S, Totoki Y, Matsushita Y, Otsuka A, Tomiyama A, et al. : Recurrent neomorphic mutations of MTOR in central nervous system and testicular germ cell tumors may be targeted for therapy. Acta Neuropathol $131: 889-901,2016$

21. Ishida M, Moore GE : The role of imprinted genes in humans. Mol Aspects Med 34 : 826-840, 2013

22. Kamakura Y, Hasegawa M, Minamoto T, Yamashita J, Fujisawa H : C-kit gene mutation: common and widely distributed in intracranial germinomas. J Neurosurg 104(3 Suppl) : 173-180, 2006 
23. Kato T, Sawamura Y, Tada M, Murata J, Abe H, Shirato H, et al. : Occult neurohypophyseal germinomas in patients presenting with central diabetes insipidus. Neurosurg Focus 5 : e6, 1998

24. Kellie SJ, Boyce H, Dunkel IJ, Diez B, Rosenblum M, Brualdi L, et al. : Intensive cisplatin and cyclophosphamide-based chemotherapy without radiotherapy for intracranial germinomas: failure of a primary chemotherapy approach. Pediatr Blood Cancer 43 : 126-133, 2004

25. Kim CY, Choi JW, Lee JY, Kim SK, Wang KC, Park SH, et al. : Intracranial growing teratoma syndrome: clinical characteristics and treatment strategy. J Neurooncol 101 : 109-115, 2011

26. Kim JW, Kim WC, Cho JH, Kim DS, Shim KW, Lyu CJ, et al. : A multimodal approach including craniospinal irradiation improves the treatment outcome of high-risk intracranial nongerminomatous germ cell tumors. Int J Radiat Oncol Biol Phys 84 : 625-631, 2012

27. Lai IC, Wong TT, Shiau CY, Hu YW, Ho DM, Chang KP, et al. : Treatment results and prognostic factors for intracranial nongerminomatous germ cell tumors: single institute experience. Childs Nerv Syst 31 : 683691, 2015

28. Matsutani M, Sano K, Takakura K, Fujimaki T, Nakamura O, Funata N, et al. : Primary intracranial germ cell tumors: a clinical analysis of 153 histologically verified cases. J Neurosurg 86 : 446-455, 1997

29. McCarthy BJ, Shibui S, Kayama T, Miyaoka E, Narita Y, Murakami M, et al. : Primary CNS germ cell tumors in Japan and the United States: an analysis of 4 tumor registries. Neuro Oncol 14 : 1194-1200, 2012

30. Mootha SL, Barkovich AJ, Grumbach MM, Edwards MS, Gitelman SE, Kaplan SL, et al. : Idiopathic hypothalamic diabetes insipidus, pituitary stalk thickening, and the occult intracranial germinoma in children and adolescents. J Clin Endocrinol Metab 82 : 1362-1367, 1997

31. Murray MJ, Bartels U, Nishikawa R, Fangusaro J, Matsutani M, Nicholson $J C$ : Consensus on the management of intracranial germ-cell tumours. Lancet Oncol 16 : e470-e477, 2015

32. Murray MJ, Horan G, Lowis S, Nicholson JC : Highlights from the Third International Central Nervous System Germ Cell Tumour symposium: laying the foundations for future consensus. Ecancermedicalscience 7 : 333,2013

33. Nakamura H, Takeshima H, Makino K, Kuratsu J : C-kit expression in germinoma: an immunohistochemistry-based study. J Neurooncol 75 : 163-167, 2005

34. Netto GJ, Nakai Y, Nakayama M, Jadallah S, Toubaji A, Nonomura N, et al. : Global DNA hypomethylation in intratubular germ cell neoplasia and seminoma, but not in nonseminomatous male germ cell tumors. Mod Pathol 21 : 1337-1344, 2008

35. Odagiri K, Omura M, Hata M, Aida N, Niwa T, Ogino I, et al. : Treatment outcomes, growth height, and neuroendocrine functions in patients with intracranial germ cell tumors treated with chemoradiation therapy. Int J Radiat Oncol Biol Phys 84 : 632-638, 2012

36. Ogiwara H, Kiyotani C, Terashima K, Morota N : Second-look surgery for intracranial germ cell tumors. Neurosurgery 76 : 658-661; discussion 661-662, 2015

37. Okada Y, Nishikawa R, Matsutani M, Louis DN : Hypomethylated X chromosome gain and rare isochromosome $12 p$ in diverse intracranial germ cell tumors. J Neuropathol Exp Neurol 61 : 531-538, 2002

38. Oosterhuis JW, Stoop H, Honecker F, Looijenga LH : Why human extragonadal germ cell tumours occur in the midline of the body: old concepts, new perspectives. Int J Androl 30 : 256-263; discussion 263-264, 2007

39. Padilla BE, Vu L, Lee H, MacKenzie T, Bratton B, O'Day M, et al. : Sacrococcygeal teratoma: late recurrence warrants long-term surveillance. Pediatr Surg Int 33 : 1189-1194, 2017

40. Phi JH, Cho BK, Kim SK, Paeng JC, Kim IO, Kim IH, et al. : Germinomas in the basal ganglia: magnetic resonance imaging classification and the prognosis. J Neurooncol 99 : 227-236, 2010

41. Phi JH, Kim SK, Lee J, Park CK, Kim IH, Ahn HS, et al. : The enigma of bifocal germ cell tumors in the suprasellar and pineal regions: synchronous lesions or metastasis? J Neurosurg Pediatr 11 : 107-114, 2013

42. Phi JH, Kim SK, Lee YA, Shin CH, Cheon JE, Kim IO, et al. : Latency of intracranial germ cell tumors and diagnosis delay. Childs Nerv Syst 29 : 1871-1881, 2013

43. Phi JH, Kim SK, Park SH, Hong SH, Wang KC, Cho BK : Immature teratomas of the central nervous system: is adjuvant therapy mandatory? J Neurosurg 103(6 Suppl) : 524-530, 2005

44. Reuter VE : Origins and molecular biology of testicular germ cell tumors. Mod Pathol 18 Suppl 2 : S51-S60, 2005

45. Richardson $B E$, Lehmann $R$ : Mechanisms guiding primordial germ cell migration: strategies from different organisms. Nat Rev Mol Cell Biol $11: 37-49,2010$

46. Rogers SJ, Mosleh-Shirazi MA, Saran FH : Radiotherapy of localised intracranial germinoma: time to sever historical ties? Lancet Oncol 6 : 509519, 2005

47. Sakuma Y, Sakurai S, Oguni S, Satoh M, Hironaka M, Saito K : c-kit gene mutations in intracranial germinomas. Cancer Sci 95 : 716-720, 2004

48. Sano K : Pathogenesis of intracranial germ cell tumors reconsidered. J Neurosurg $90: 258-264,1999$

49. Sawamura $Y$, Ikeda J, Shirato $H$, Tada M, Abe H : Germ cell tumours of the central nervous system: treatment consideration based on 111 cases and their long-term clinical outcomes. Eur J Cancer 34 : 104-110, 1998

50. Sette C, Dolci S, Geremia R, Rossi P: The role of stem cell factor and of alternative c-kit gene products in the establishment, maintenance and function of germ cells. Int J Dev Biol 44 : 599-608, 2000

51. Sheikine Y, Genega E, Melamed J, Lee P, Reuter VE, Ye H : Molecular genetics of testicular germ cell tumors. Am J Cancer Res 2 : 153-167, 2012

52. Sukov WR, Cheville JC, Giannini C, Carlson AW, Shearer BM, Sinnwell JP, et al. : Isochromosome $12 p$ and polysomy 12 in primary central nervous system germ cell tumors: frequency and association with clinicopathologic features. Hum Pathol 41 : 232-238, 2010

53. Tanabe M, Mizushima M, Anno Y, Kondou S, Dejima S, Hirao DJ, et al. : Intracranial germinoma with Down's syndrome: a case report and review of the literature. Surg Neurol $47: 28-31,1997$

54. Teilum G, Albrechtsen R, Norgaard-Pedersen B : The histogeneticembryologic basis for reappearance of alpha-fetoprotein in endodermal sinus tumors (yolk sac tumors) and teratomas. Acta Pathol Microbiol Scand A $83: 80-86,1975$

55. Tian Q, Frierson HF Jr, Krystal GW, Moskaluk CA : Activating c-kit gene 
mutations in human germ cell tumors. Am J Pathol 154 : 1643-1647, 1999

56. Trama A, Berrino $F$ : The epidemiology of malignant germ cell tumors: the EUROCARE study in Nogales FF, Jimenez RE (eds) : Pathology and Biology of Human Germ Cell Tumors. Germany : Springer-Verlag $\mathrm{GmbH}, 2017$, pp11-21

57. Wang L, Yamaguchi S, Burstein MD, Terashima K, Chang K, Ng HK, et al. : Novel somatic and germline mutations in intracranial germ cell tumours. Nature 511 : 241-245, 2014

58. Weiner HL, Lichtenbaum RA, Wisoff JH, Snow RB, Souweidane MM, Bruce
JN, et al. : Delayed surgical resection of central nervous system germ cell tumors. Neurosurgery 50 : 727-733; discussion 733-734, 2002

59. Wolf SS, Patchev VK, Obendorf $\mathrm{M}:$ A novel variant of the putative demethylase gene, s-JMJD1C, is a coactivator of the AR. Arch Biochem Biophys 460 : 56-66, 2007

60. Yoshida M, Matsuoka K, Nakazawa A, Yoshida M, Inoue T, Kishimoto $H$, et al. : Sacrococcygeal yolk sac tumor developing after teratoma: a clinicopathological study of pediatric sacrococcygeal germ cell tumors and a proposal of the pathogenesis of sacrococcygeal yolk sac tumors. J Pediatr Surg 48 : 776-781, 2013 